\title{
Collective Correlations in Spherical Nuclei and the Structure of Giant Resonances*
}

\author{
Dieter Drechsel $\dagger$ \\ National Bureau of Standards, Washington, D. C. \\ AND \\ J. B. SEABorn $\ddagger$ AND WALter Greiner \\ Institut für Theoretische Physik der Universität, Frankfurt/Main, Germany
}

(Received 14 April 1967)

\begin{abstract}
The theory of collective correlations in nuclei is formulated for giant resonances interacting with surface vibrations. The giant dipole states are treated in the particle-hole framework, while the surface vibrations are described by the collective model. Consequently, this treatment of nuclear structure goes beyond both the common particle-hole model (including its various improvements which take ground-state correlations into account) and the pure collective model. The interaction between giant resonances and surface degrees of freedom as known from the dynamic collective theory is formulated in the particle-hole language. Therefore, the theory contains the particle-hole structures and the most important "collective intermediate" structures of giant resonances. Detailed calculations are performed for ${ }^{12} \mathrm{C},{ }^{28} \mathrm{Si}$, and ${ }^{60} \mathrm{Ni}$. A good detailed agreement between theory and experiment is obtained for all these nuclei, although only ${ }^{60} \mathrm{Ni}$ is in the region where one would expect the theory to work well $(50<A<110)$.
\end{abstract}

\section{INTRODUCTION}

$\mathrm{C}$ OLLECTIVE nuclear states have been investigated quite extensively during the past ten years within the framework of the collective model, as well as in terms of various microscopic approaches. ${ }^{1}$ While the former model has the advantage of being lucid, the latter has the advantage of being more detailed in that special shell-model features are more fully described microscopically.

However, in the comparison of the theoretical results with the experimental $\gamma$-absorption cross sections, evidently neither of these approaches is complete. In fact, the particle-hole calculations for light and heavy magic nuclei explain only gross features of giant resonances, such as the existence of one or two states shifted up in energy which carry an appreciable part of the dipole strength. We may call this the doorway structure. For nearly all nuclei, however, it is known that the giant resonances show much additional structure. Such structure may be divided into two groups: (a) the main substructure, which we call collective intermediate structure-by this we mean that the giant resonance splits into three, four, or more main distinct resonances because of their interaction with other collective degrees of freedom such as the surface vibrations; (b) on top of this collective structure, we may find a small substructure which we call noncollective structure.

In ${ }^{16} \mathrm{O}$, for example, the two main resonances at 22 and $24.0 \mathrm{MeV}$ (see Fig. 1) are in this sense doorway

${ }^{*}$ Work supported by the Deutsche Forschungsgemeinschaft with a contract for studies in nuclear structure and by the Bundesministerium für Wissenschaft und Forschung.

$\dagger$ On leave of absence from Universität Frankfurt, Main, Germany.

$\ddagger$ Present address: Department of Physics, North Texas State University, Denton, Texas.

${ }^{1}$ As a general review of giant-resonance physics see $M$. Danos and E. G. Fuller, Ann. Rev. Nucl. Sci. 15, 28 (1965); V. Shevchenko and N. Yudin, At. Energy Rev. 3,3 (1965). structures, because both levels correspond to different $1 p$ - $1 h$ configurations. Their main substructure, i.e., the peak at $22 \mathrm{MeV}$ and the resonance at $23 \mathrm{MeV}$, as well as the three peaks between 24.0 and $26.5 \mathrm{MeV}$, are collective intermediate structures. The remaining noncollective fine structure is small for the total $\gamma$-absorption cross section but stands out more clearly in reactions like $(p, \gamma)$.

One may summarize the success of the various theoretical approaches as follows: The particle-hole model has been successful in explaining the doorway structure. The calculations of Elliot and Flowers and others ${ }^{2-5}$ explained just this kind of giant-resonance structure. The dynamic collective theory, ${ }^{6-10}$ on the other hand, explains the collective intermediate structure for medium and heavy nuclei. This has been shown in an exciting development, both in theory and experiment, during the last few years. ${ }^{11-13}$

It therefore seems to be worthwhile and, in fact,

${ }^{2}$ J. P. Elliot and B. H. Flowers, Proc. Roy. Soc. (London) A242, 57 (1957).

${ }^{3}$ S. Fallieros, R. A. Ferrell, and M. K. Pal, Phys. 15, 363 (1960).

${ }^{4}$ G. E. Brown and M. Bolsterli, Phys. Rev. Letters 3, 472 (1959).

5 V. Gillet, Ph.D. dissertation, University of Paris, 1962 (unpublished).

${ }_{6}^{6}$ M. Danos and W. Greiner, Phys. Rev. 134, B284 (1964).

${ }^{7}$ M. Danos, W. Greiner, and C. B. Kohr, Phys. Rev. 138, B1055 (1965).

${ }^{8}$ J. Le Tourneux, Kgl. Danske Videnskab. Selskab. Mat. Fys. Medd. 34, 11 (1965).

${ }_{9}$ M. G. Huber, H. J. Weber, M. Danos, and W. Greiner, Phys. Rev. Letters 15, 529 (1965); Phys. Rev. 155, 1073 (1967).

${ }^{10} \mathrm{H}$. J. Weher, M. G. Huber, and W. Greiner, Z. Physik 190, 25 (1966); 190, 25 (1966); 192, 182 (1966); 192, 223 (1966).

${ }^{11}$ D. S. Fielder, J. Le Tourneux, K. Min, and W. D. Whitehead, Phys. Rev. Letters 15, 33 (1965).

${ }^{12}$ S. Costa, F. Ferrero, C. Manfredotti, L. Pasqualini, G. Pieragino, P. G. Rama, and H. Arenhövel, Nuovo Cimento 48, 460 (1967).

${ }^{13}$ D. V. Webb, B. M. Spicer, and H. Arenhövel (to be published). 


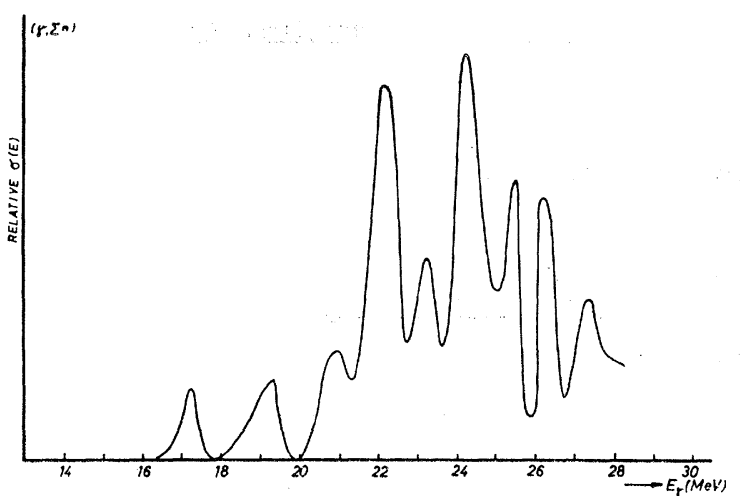

FIG. 1. $\left(\gamma, \sum n\right)$ cross section of $\mathrm{O}^{16}$.

necessary to combine the collective and the particle-hole approach in order to describe both the doorway and the collective structure. The main purpose of this paper is to develop such a theory.

A few words should be said to answer the question which immediately comes to mind at this stage: Would not a particle-hole calculation, if performed in the full Hilbert space, i.e., a diagonalization in the basis of many-particle-many-hole configurations, contain everything? Of course, it would. In the first place, however, it is not satisfying to obtain results from the diagonalization of a giant matrix, and a more physical approach seems necessary to get insight into the structure and the dynamics of the nucleus. Secondly, even if one would like to do so, it is impossible to carry out such calculations because of the tremendous number of many-particle-many-hole configurations that would have to be included. (See, for example, the work of Boeker. ${ }^{14}$ )

We now come to the specific contents of this paper. In Sec. II we give a microscopic outline of the idea of the collective correlations. The various structures introduced above in a somewhat phenomenological way are depicted by graphs. Also, an interpretation of the interaction of the giant resonances with other collective degrees of freedom in terms of the many-particle-manyhole configuration matrix is given there. Section III contains a brief review of the dynamic collective theory, which is necessary for the understanding and explicit formulation of the idea of collective correlations, presented in Sec. IV. The complete Hamiltonian containing collective correlations is discussed in Sec. V, which also

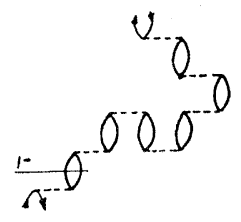

FIG. 2. $1 p-1 h$ configuration.

${ }^{14}$ E. Boeker, W. M. De Mujnick, and C. C. Jonker, in Comptes Rendus du Congrès International de Physique Nucléaire, edited by $\mathrm{P}$. Gungenberger (Centre National de la Recherche Scientifique, Paris, 1964), Vol. II, p. 405.

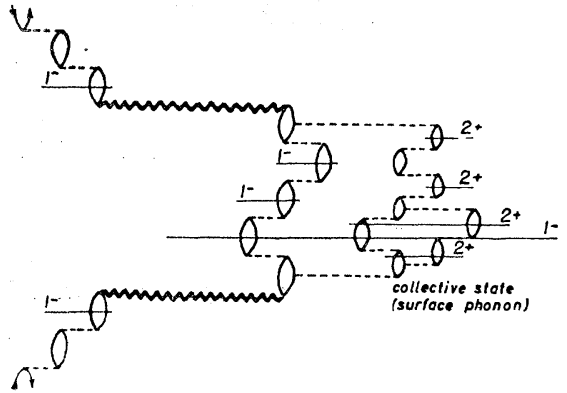

Frg. 3. The interaction of a collective $1 p-1 h$ state (giant dipole resonance) with a collective more-particle-more-hole state [(giant dipole $)+$ (surface quadrupole phonon) $]$.

contains the classification of the basis states and the setup of the configuration matrix. Finally, in Sec. VI, we compare detailed calculations with experiments and discuss the various results.

\section{MICROSCOPIC DESCRIPTION OF COLLECTIVE CORRELATIONS}

All collective modes contain a large amount of singleparticle excitation, i.e., they are predominantly linear combinations of states which differ from the ground state in the state of one particle only. In other words, they are essentially $1 p-1 h$ excitations. This must necessarily be so because they have large electromagnetic transition probabilities to the ground state and the transition operator is a sum of one-body operators. In terms of graphs these $1 p-1 h$ components of collective states are thus represented by single "sausages" which may go backward as well as forward (Fig. 2). In such chains each particle and its hole partner are coupled to the spin and parity of the collective state, e.g., $1^{-}$for the dipole state, $2^{+}$for surface oscillations. It has been shown earlier ${ }^{6-10}$ that giant resonance states and surface states are strongly coupled. The reason for this is the coherent structure of the collective states. Assuming that the matrix elements between the various $p-h$ states have the same sign (e.g., as in a schematic model), one immediately gets the strong correlations which are predicted by the collective theory. ${ }^{15}$ Such a state would be depicted in graphs like Fig. 3. The sausages at the right-hand side represent the surface vibration consisting of $1 p-1 h, 2 p-2 h$, etc., components which is coupled with a particle-hole excitation to $1^{-}$. The strong wave lines in Fig. 3 represent the strong coupling between these particular $1^{-}$ collective states. The configurations corresponding to the region of Fig. 3, where two sausages are present, do not have multipole moments to the ground state. The transition strength of such a state is thus decreased and reappears at the state which, in the limit of small

\footnotetext{
${ }^{15} \mathrm{We}$ are very grateful to C. A. Levinson for bringing this to
} our attention. 


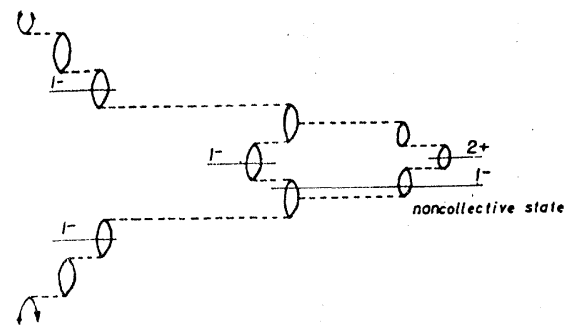

FIG. 4. The interaction of a collective $1 p-1 h$ state with a noncollective more-particle-more-hole configuration. The difference from the graph in Fig. 3 is the weak interaction of the left-hand and right-hand bubbles.

coupling, consists of a surface quantum in addition to the dipole state.

The graph of Fig. 3 has to be distinguished from a graph as shown in Fig. 4. The latter represents the coupling of the giant resonance to a noncollective $2^{+}$ state, which is indicated simply by dashed connections between the coupled $\left(2^{+}-1^{-}\right)$bubbles and the pure $1^{-}$ chain.

Since the matrix elements between the $1^{-}$and the collective $\left(2^{+}-1^{-}\right)$states of Fig. 3 are very strong, such graphs lead to the main structure of the giant resonances (the collective intermediate structure), while graphs as shown in Fig. 4 give only noncollective fine structure. As mentioned earlier, the latter shows up in experiments as small additional substructure of the main collective structure. The $1 p-1 h$ or doorway structure is given by different graphs of the type shown in Fig. 2. We are therefore led to the hierarchy shown in Fig. 5. The strong matrix elements between the two (or in general more) collective chains in Fig. 3 represent the collective correlations. The two collective states interact strongly, and these correlations lead to the collective intermediate structure.

Note that this hierarchy of essentially three different types of structure is different from the usual one where the classification is according to $1 p-1 h, 2 p-2 h$, etc., configurations. The first of these, the $p-h$ configurations, are identical with the doorway structure. The collective and noncollective configurations, however, are compli-

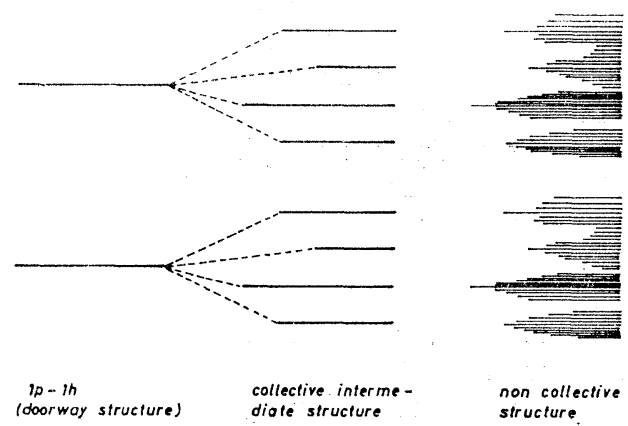

FIG. 5. Schematic representation of doorway $1 p-1 h$, collective intermediate, and noncollective structure.

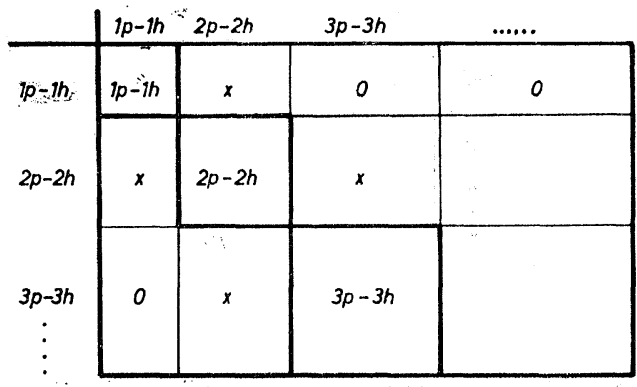

FIG. 6. The total configuration matrix of the particle-hole Hamiltonian.

cated superpositions of $1 p-1 h, 2 p-2 h, 3 p-3 h$, etc., configurations. They are only classified according to the magnitude of their coupling strength to the doorway $1 p-1 h$ structure.

Now let us look at the complete configuration matrix of the particle-hole Hamiltonian and interpret the hierarchy of Fig. 5 in terms of the configuration matrix. In Fig. 6 the total configuration matrix is shown schematically. In ordinary $p-h$ calculations, only the $1 p-1 h$ submatrix is considered. The higher configurations are completely neglected. In fact, taking all the higher configurations into account increases extremely the size of the matrix. For ${ }^{16} \mathrm{O}$, Boeker ${ }^{14}$ estimated about $5002 p-2 h$ states up to $3 \hbar \omega$ excitation energy. Nevertheless, we can perform the following Gedankenexperiment: Suppose we prediagonalize the $2 p-2 h, 3 p-3 h$, etc., part of the matrix and denote the resulting states by $\varphi_{1}, \varphi_{2}$, $\varphi_{3}$, etc. The $\varphi_{i}$ 's are complicated superpositions of many-particle-many-hole configurations, and the total configuration matrix is shown schematically in Fig. 7. There now occur only matrix elements between the states $\varphi_{i}$ and the $1 p-1 h$ states and, of course, within the $1 p-1 h$ submatrix. Some of these matrix elements are very strong and are indicated by large crosses in Fig. 7. Such states $\varphi_{i}$ (in Fig. 7 they are $\varphi_{2}$ and $\varphi_{4}$ ) are identified with collective states which correspond, for example, to the region in Fig. 3 where two bubble chains exist. The other states $\varphi_{1}, \varphi_{3}, \varphi_{5}$ in Fig. 7) correspond to the same region in Fig. 4. The only difference is that the former are collective ones with strong matrix elements to the $1 p-1 h$ states giving the main structure of the giant resonance, while the latter are of noncollective type and have only weak coupling with the $1 p-1 h$ subspace. Therefore, we are interested mainly in the collective states (Fig. 3). We can not treat them with all their microscopic structure. This would imply that we are able to prediagonalize the $2 p-2 h, 3 p-3 h$, etc., subspace. We will describe these states in the collective model, i.e., as states where $1 p-1 h$ configurations are coupled to surface phonons. Of course, there arises immediately the difficulty of finding the strong matrix elements of these states to the $1 p-1 h$ submatrix (Fig. 7). This problem will be solved in Sec. IV, where we "translate" the interaction between dipole states and 


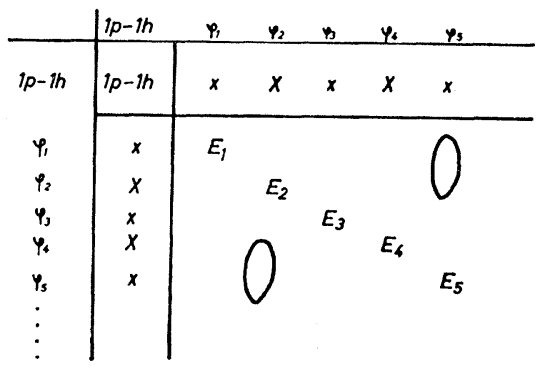

FIG. 7. Configuration matrix after prediagonalization of the $2 p-2 h, 3 p-3 h$, etc., subspace.

surface vibrations obtained in the collective model into the particle-hole language.

\section{REVIEW OF THE DYNAMIC COLLECTIVE THEORY}

The dynamic collective theory for spherical nuclei ${ }^{8-10}$ investigates the following Hamiltonian:

where

$$
H=H_{\text {quad }}+H_{\text {dip }}+H_{\text {dip quad }},
$$

$$
\begin{aligned}
H_{\text {quad }}=\left(\frac{1}{2} \sqrt{ } 5\right) B_{2}\left[\dot{\alpha}^{[2]} \times \dot{\alpha}^{[2]}\right]^{[0]} & \\
& +\left(\frac{1}{2} \sqrt{ } 5\right) C_{2}\left[\alpha^{[2]} \times \alpha^{[2]}\right]^{[0]}
\end{aligned}
$$

describes the nuclear surface vibrations in the harmonic approximation. This is, of course, no restriction, and it is straightforward to include anharmonic terms as well. The operator $\alpha^{[2]}$ is the tensor of rank 2 and positive parity for the surface quadrupole collective variables. The dipole part of (1) is

$$
H_{\text {dip }}=-\frac{1}{2} \sqrt{3} B_{1}\left[\dot{\alpha}^{[1]} \times \dot{\alpha}^{[1]}\right]^{[0]}-\frac{1}{2} \sqrt{3} C_{1}\left[\alpha^{[1]} \times \alpha^{[1]}\right]^{[0]},
$$

where $\alpha^{[1]}$ is the tensor of rank 1 and negative parity describing the collective variables of the giant dipole resonances. The giant resonances are considered as fluctuations of the proton and neutron densities, $\rho_{p}(\mathbf{r}, t)$ and $\rho_{n}(\mathbf{r}, t)$,

$$
\begin{aligned}
& \rho_{p}(\mathbf{r}, t)=\rho_{p}(0)+\eta(\mathbf{r}, t), \\
& \rho_{n}(\mathbf{r}, t)=\rho_{n}(0)-\eta(\mathbf{r}, t),
\end{aligned}
$$

where incompressibility of nuclear matter is assumed. For dipole fluctuations, one has

if

$$
\eta(\mathbf{r}, t)=-F \sqrt{3} j_{1}\left(k_{0} r\right)\left[\alpha^{[1]} \times Y^{[1]}\right]^{[0]},
$$

$$
r \leqslant R(\vartheta, \varphi)=R_{0}\left\{1+(\sqrt{ } 5)\left[\alpha^{[2]} \times Y^{[2]}\right]^{[0]}\right\} .
$$

Here $F$ is a normalization constant, $R_{0}$ is the nuclear equilibrium radius, and $k_{0}$ is the wave number of the dipole oscillations. The time dependence is contained in $\alpha^{[1]}$. The interaction of giant dipole resonances and surface degrees of freedom is ${ }^{16}$

$$
\begin{aligned}
H_{\mathrm{dip} \mathrm{quad}}= & K_{1}\left[\left[\alpha^{[1]} \times \alpha^{[1]}\right]^{[2]} \times \alpha^{[2]}\right]^{[0]} \\
& +K_{20}\left[\alpha^{[1]} \times \alpha^{[1]}\right]^{[0]}\left[\alpha^{[2]} \times \alpha^{[2]}\right]^{[0]} \\
& +K_{22}\left[\left[\alpha^{[1]} \times \alpha^{[1]}\right]^{[2]} \times\left[\alpha^{[2]} \times \alpha^{[2]}\right]^{[2]}\right]^{[0]},
\end{aligned}
$$

${ }^{16}$ T. Urbas and W. Greiner, Z. Physik 196, 44 (1966). where the coupling constants $K_{1}, K_{20}$, and $K_{22}$ have been calculated in the hydrodynamic model ${ }^{16}$ :

$K_{1}=-1.588 C_{1}, K_{20}=-0.708 C_{1}, K_{22}=-0.936 C_{1}$. (7)

$C_{1}$ is the same parameter as in (3) and can be expressed in terms of the asymmetry energy parameter $\kappa$ of the Bethe-Weizsäcker mass formula,

$$
C_{1}=8 \kappa Z^{2} / A \text {. }
$$

The Hamiltonian (1) is diagonalized in the basis which is constructed by coupling one dipole phonon ${ }^{17}$ and $N$ quadrupole phonons to $1^{-}$;

$$
\left|N_{1} l_{1} ; N_{2} l_{2} v \alpha \| I M\right\rangle \text {. }
$$

Here $N_{1}=1$ and $N_{2} \leqslant N$ are the number of dipole and quadrupole phonons, respectively. The corresponding angular momenta are $l_{1}=1$ and $l_{2}, v$ is the seniority quantum number and $\alpha$ denotes additional quantum numbers of the surface phonon states.

The dipole operator is easily obtained as

$$
D_{\mathrm{coll}}^{[1]}=\left(\frac{4 \pi}{3}\right)^{1 / 2} \int_{\text {nucleus }} \eta(\mathbf{r}) r Y^{[1]} d \tau
$$

and is explicitly given by

$$
D_{\mathrm{coll}}{ }^{[1]}=M_{0}\left[\alpha^{[1]}+M_{1}\left[\alpha^{[1]} \times \alpha^{[2]}\right]^{[1]}+\cdots\right],
$$

where

$$
M_{0}=\frac{R_{0} Z}{x_{0}}\left(\frac{2}{x_{0}^{2}-2}\right)^{1 / 2}, \quad M_{1}=-\frac{x_{0}^{2}}{2 \pi}, \quad x_{0}=k_{0} R_{0}=2.08 \text {, }
$$

and $R_{0}$ is the nuclear radius. Later we will need the inverse of (11), i.e.,

$$
\alpha^{[1]}=\frac{1}{M_{0}} D_{\mathrm{coll}}^{[1]}-\frac{M_{1}}{M_{0}}\left[D_{\mathrm{coll}}^{[1]} \times \alpha^{[2]}\right]^{[1]}+\cdots
$$

The results of the dynamic collective theory for medium and heavy nuclei show that the main structure of the giant resonances is given by the strong coupling of giant resonances to surface vibrations, ${ }^{9,10,12}$ the matrix elements being of the order of $2 \mathrm{MeV}$. In fact, if we neglect the dipole-quadrupole coupling we are led to only one single $1^{-}$state with dipole strength. This occurs because the collective model describes only the dominant dipole state and neglects all the other $1 p-1 h$ states with less dipole strength. For example, the $22-\mathrm{MeV}$ giant resonance in $\mathrm{O}^{16}$ is to be identified with the one-dipole-phonon state of the collective model. The $24.5-\mathrm{MeV}$ giant resonance is an additional $1 p-1 h$ configuration which has no corresponding state in the collective model. It is therefore necessary to extend the Hamiltonian (1) along the lines suggested in Sec. II. This will be done in the following section.

\footnotetext{
${ }^{17}$ The three-dipole phonon states are treated by perturbation theory.
} 


\section{SEMIMICROSCOPIC FORMULATION OF COLLECTIVE CORRELATIONS}

The dipole giant resonances obviously interact very strongly with surface vibrations. Therefore, the most important states leading to collective intermediate structure for the giant resonances will be such nuclear states where a $(1 p-1 h)^{1^{-}}$configuration is coupled to surface excitations. We still describe the latter in the collective model, i.e., in the phonon approximation, for simplicity. ${ }^{18}$ It seems, therefore, most natural to generalize the Hamiltonian (1) in the following way ${ }^{19}$ :

(a) $H_{\text {dip }}$ is replaced by $H_{\text {ph }}{ }^{(1)}$;

$$
H_{\mathrm{dip}} \Rightarrow H_{\mathrm{ph}}^{(1)} \text {, }
$$

where $H_{\mathrm{ph}}{ }^{(1)}$ stands for the particle-hole Hamiltonian in the $(1 p-1 h)^{1^{-}}$subspace (see Fig. 7).

(b) The interaction (6) between surface vibrations and the dipole states has to be interpreted in the particlehole language. This is achieved by the requirement that the dipole operators in the two pictures are the same, i.e.,

where

$$
D_{\text {coll }}^{[1]}=D_{\text {ph }^{[1]}},
$$

$$
D_{\mathrm{ph}}^{[1]}=\left(\frac{4 \pi}{3}\right)^{1 / 2} \sum_{\kappa_{1}, \kappa_{2}}\left\langle\kappa_{1}\left|\frac{1}{2} \tau_{3} r Y^{[1]}\right| \kappa_{2}\right\rangle a_{\kappa_{1}}^{\dagger} a_{\kappa_{2}} .
$$

Inserting (13) and (11) in (6), we obtain for the interaction

$$
\begin{aligned}
H_{\mathrm{di} \rho \text { quad }} & \Rightarrow H_{\mathrm{ph}, \mathrm{quad}}=\kappa_{1}\left[\left[D_{\mathrm{ph}}^{[1]} \times D_{\mathrm{ph}}{ }^{[1]}\right]^{[2]} \times \alpha^{[2]}\right]^{[0]} \\
& +\kappa_{20}\left[D_{\mathrm{ph}}{ }^{[1]} \times D_{\mathrm{ph}^{[1]}}\right]^{[0]}\left[\alpha^{[2]} \times \alpha^{[21}\right]^{[0]} \\
& +\kappa_{22}\left[\left[D_{\mathrm{ph}}^{[1]} \times D_{\mathrm{ph}^{[1]}}\right]^{[2]} \times\left[\alpha^{[2]} \times \alpha^{[2]}\right]^{[2]}\right]^{[0]}
\end{aligned}
$$

where

$$
\begin{gathered}
\kappa_{1}=-1.588 \times 4 x_{0}^{2}\left(x_{0}^{2}-2\right) \kappa / A R_{0}^{2}=-64 \kappa / A R_{0}^{2}, \\
\kappa_{20}=-\left(0.708+1.588(2 / 5 \pi)^{1 / 2} x_{0}^{2}\right) \\
\quad \times 4 x_{0}^{2}\left(x_{0}^{2}-2\right) \kappa / A R_{0}^{2}=-127.5 \kappa / A R_{0}^{2}, \\
\kappa_{22}=-\left(0.936+1.588(7 / 10 \pi)^{1 / 2} x_{0}^{2}\right) \\
\quad \times 4 x_{0}^{2}\left(x_{0}^{2}-2\right) \kappa / A R_{0}^{2}=-169 \kappa / A R_{0}^{2} .
\end{gathered}
$$

Here $\kappa$ is the symmetry energy constant of the BetheWeizsäcker mass formula. The renormalization of the coupling constants $K_{1}, K_{20}$, and $K_{22}$ occurring in (6) to the values $\kappa_{1}, \kappa_{20}$, and $\kappa_{22}$ in Eq. (16) is due to the second term of (11). The physical origin of this additional term in (11) is quite interesting. It takes into account the change of the single-particle functions (computed in a spherical well) due to the dynamic surface vibrations. In fact, the potential well is oscillating about a spherical equilibrium value. The singleparticle functions $\psi_{j m}$ depend, therefore, on the surface

\footnotetext{
${ }^{18}$ At least in medium and heavy nuclei these $2^{+}$states are very complicated superpositions of various many-particle-many-hole states.

${ }_{19}$ D. Drechsel, J. B. Seaborn, and W. Greiner, Phys. Rev. Letters 17, 488 (1966).
}

variables $\alpha^{[2]}$, i.e.,

$$
\psi_{j m}=\psi_{j m}(r, \alpha)=\psi_{j m}(r, 0)+\partial \psi_{j m} /\left.\partial \alpha\right|_{\alpha=0 \alpha} .
$$

The term proportional to $\alpha$ leads to a similar term in the transition charges $\eta(\mathbf{r}, t)$ given by the collective model (5). Therefore, by keeping the second term of (11) we take into account additional corrections for the singleparticle wave functions due to the dynamic deformation of the shell-model potential.

The full semimicroscopic Hamiltonian for giant resonances is now

$$
H=H_{\mathrm{ph}}^{(1)}+H_{\mathrm{quad}}+H_{\mathrm{ph}, \mathrm{quad}} .
$$

$H_{\mathrm{ph} \text {,quad }}$ describes the strong matrix elements shown in Fig. 7 between the collective many-particle-many-hole configurations and the $1 p-1 h$ states. Formally, it has the structure of an additional two-body force between the particle-hole states which, however, depends on the surface collective coordinates $\alpha^{[2]}$. This interaction leads to the collective correlations between the giant resonances and the surface vibrations.

Expressed in microscopic terminology, the matrix elements between two states are large if these two states are essentially coherent superpositions of $1 p-1 h$ configurations or products of such superpositions, i.e., if they are collective states. Therefore, the matrix elements shown in Figs. 3 and 4 are essentially proportional to the dipole moment in the initial state and the dipole and quadrupole moments in the intermediate state. Thus they are strong only in the case of Fig. 3.

\section{DIAGONALIZATION OF THE INTERACTION BETWEEN THE $(1 p-1 h)^{1}$ STATES}

The eigenstates and eigenvalues of the Hamiltonian (17) are found by diagonalization of the energy matrix. The basis for the matrix is formed by the pure $(1 p-1 h)$ states with $0,1,2$, etc., phonons excited:

$$
\left|\psi_{n}\right\rangle=\left|j_{h} l_{h} n_{h}, j_{p} l_{p} n_{p}, J ; N l v \alpha ; 1^{-}\right\rangle,
$$

where $j, l$, and $n$ are, respectively, the total-angularmomentum, orbital-angular-momentum, and radial quantum numbers of the single-particle states. The $1 p-1 h$ configuration is coupled to the intermediate angular momentum $J . N$ stands for the number of phonons, $v$ for the seniority, and $l$ for the angular momentum of the phonon wave function. The total spin of the states considered is unity, and their parity is negative.

For numerical reasons we found it useful to prediagonalize the $1 p-1 h$ subspace with the residual interaction included. ${ }^{20}$ This gives the gross distribution of the dipole strength (doorway structure). We then take into account the collective correlations, $H_{\mathrm{ph} \text {,quad }}$. Since the matrix elements of the collective correlations (15) between two states are essentially proportional to the

${ }^{20}$ J. B. Seaborn, D. Drechsel, and W. Greiner, Z. Physik 202, 32 (1967). 
product of the dipole strengths of the states involved, we are allowed to omit such prediagonalized $1 p-1 h$ states which are far off the giant resonance and carry only a small amount of dipole strength. In this way we can keep the matrix which is to be diagonalized relatively small.

The matrix elements of the three terms of $H_{\mathrm{ph}, \mathrm{quad}}$ between the basis states are

$$
\left\langle J^{\prime}, l^{\prime} ; I\left|\left[\alpha^{[2]} \times\left[D_{\mathrm{ph}}^{[1]} \times D_{\mathrm{ph}}^{[1]}\right]^{[2]}\right]^{[0]}\right| J, l ; I\right\rangle=\frac{1}{\sqrt{ } 5}(-)^{J+l^{\prime}+I}\left\{\begin{array}{lll}
I & l^{\prime} & J^{\prime} \\
2 & J & l
\end{array}\right\}\left\langle J^{\prime}\left\|\left[D_{\mathrm{ph}}^{[1]} \times D_{\mathrm{ph}^{[1]}}\right]^{[2]}\right\| J\right\rangle\left\langle l^{\prime}\left\|\alpha^{[2]}\right\| l\right\rangle
$$

and

$$
\begin{aligned}
\left\langle J^{\prime}, l^{\prime} ; I\right|\left[\left[\alpha^{[2]} \times \alpha^{[2]}\right]^{[K]} \times\right. & {\left.\left[D_{\mathrm{ph}}{ }^{[1]} \times D_{\mathrm{ph}}{ }^{[1]}\right]^{[K]}\right]^{[0]}|J, l ; I\rangle } \\
& =\frac{1}{(2 K+1)^{1 / 2}}(-)^{J+l^{\prime}+I}\left\{\begin{array}{lll}
I & l^{\prime} & J^{\prime} \\
K & J & l
\end{array}\right\}\left\langle J^{\prime}\left\|\left[D_{\mathrm{ph}}{ }^{[1]} \times D_{\mathrm{ph}}{ }^{[1]}\right]^{[K]}\right\| J\right\rangle\left\langle l^{\prime}\left\|\left[\alpha^{[2]} \times \alpha^{[2]}\right]^{[K]}\right\| l\right\rangle,
\end{aligned}
$$

where $K=0$ or 2 . The reduced matrix elements of the surface quadrupole amplitudes $\alpha^{[2]}$ are given in detail elsewhere. ${ }^{10}$ The reduced matrix elements of the dipole operators are

$$
\begin{aligned}
\left\langle J_{3}{ }^{4} \|\right. & {\left.\left.\left[D_{\mathrm{ph}}{ }^{[1]} \times D_{\mathrm{ph}}{ }^{[1]}\right]\right]^{[K]} \| J_{1}{ }^{2}\right\rangle=\frac{1}{2} \hat{l}_{1} \hat{l}_{2} \hat{l}_{3} \hat{l}_{4} \hat{\jmath}_{1} \hat{\jmath}_{2} \hat{\jmath}_{3} \hat{\jmath}_{4} \hat{J} \hat{K} \hat{J}^{\prime} } \\
& \times\left[(-)^{j_{2}+j_{4}} \delta_{J J^{\prime}} \delta_{J^{\prime}} 1^{1}-\left(\begin{array}{lll}
l_{1} & l_{2} & 1 \\
0 & 0 & 0
\end{array}\right)\left(\begin{array}{lll}
l_{3} & l_{4} & 1 \\
0 & 0 & 0
\end{array}\right)\left\{\begin{array}{lll}
l_{1} & j_{1} & \frac{1}{2} \\
j_{2} & l_{2} & 1
\end{array}\right\}\left\{\begin{array}{lll}
l_{3} & j_{3} & \frac{1}{2} \\
j_{4} & l_{4} & 1
\end{array}\right\}\right. \\
& \times R^{12} R^{34}+(-)^{l_{3}+l_{4}+J} \delta_{\tau_{2} \tau}\left(\begin{array}{lll}
l_{1} & l_{3} & 1 \\
0 & 0 & 0
\end{array}\right)\left(\begin{array}{lll}
l_{2} & l_{4} & 1 \\
0 & 0 & 0
\end{array}\right) \\
& \left.\times \sum_{L L^{\prime} S}(-)^{L+S}(2 L+1)\left(2 L^{\prime}+1\right)(2 S+1)\left\{\begin{array}{rrr}
K & L & L^{\prime} \\
S & J^{\prime} & J
\end{array}\right\}\left\{\begin{array}{lll}
l_{1} & l_{3} & 1 \\
l_{2} & l_{4} & 1 \\
L & L^{\prime} & K
\end{array}\right\}\left\{\begin{array}{lll}
l_{1} & \frac{1}{2} & j_{1} \\
l_{2} & \frac{1}{2} & j_{2} \\
L & S & J
\end{array}\right\}\left\{\begin{array}{lll}
l_{3} & \frac{1}{2} & j_{3} \\
l_{4} & \frac{1}{2} & j_{4} \\
L^{\prime} & S & J^{\prime}
\end{array}\right\} R^{13} R^{24}\right] .
\end{aligned}
$$

Here the indices 1 and 3 correspond to holes and the indices 2 and 4 to particles and $\hat{l}_{i}=\left(2 l_{i}+1\right)^{1 / 2}$. The radial integrals $R^{i f}$ are given by

$$
R^{i f}=\int_{0}^{\infty} R_{n_{i} l_{i}}(r) r R_{n f} l_{f}(r) r^{2} d r .
$$

Evidently the direct term is essentially the product of the dipole moments of the particle-hole states. This is, of course, in complete analogy to the results of the collective model. ${ }^{9,10}$ The direct term exists only for the case where both $1 p-1 h$ configurations are coupled to $J=1$ and negative parity. The second term of (21) is an exchange term. The two particles and two holes are separately coupled to a $1^{-}$state. This term vanishes for double magic nuclei, provided that $3 \hbar \omega$ and higher excitations are discarded. For nonmagic nuclei, the exchange term mixes $(1 p-1 h)^{1-}$ states with $(1 p-1 h)^{2^{-}}$and $(1 p-1 h)^{3^{-}}$states. The latter ones are, of course, always coupled with vibrational wave functions to total angular momentum and parity $1^{-}$. In practical calcula- tions, however, this effect turned out to be small because of recoupling coefficients. Neglecting the exchange term, the matrix element between the two $1 p-1 h$ configurations will be proportional to the geometric mean of the dipole strength in the two states. Thus the mixing between a pure particle-hole state and its first vibrational satellite (i.e., the same $1 p-1 h$ state with one surface phonon excited) is proportional to the dipole strength of the pure $1 p-1 / h$ configuration. The situation is, however, much more complex than in the dynamic collective theory. Even in the case without collective correlations, we now have more $1 p-1 h$ states, with some dipole strength. Superimposed on these we have a spectrum of one or more additional phonons.

In the present calculations we have used harmonic oscillator wave functions for the radial wave functions $R_{n l}$. Once we have obtained the eigenvalues $E_{n}$ and corresponding eigenvectors by diagonalization in the configuration space consisting of $(1 p-1 h)^{1^{-}}$states and up-to- $N$-phonon states, the integrated photoabsorption cross section $\sigma_{n}$ is given by

$$
\sigma_{n}=4 \pi^{2} \alpha E_{n}\left|\left(\sum_{i} a_{i}\left\langle h_{i} p_{i} 1 ; N=v=l=0 ; 1-\left|D_{\mathrm{ph}^{[1]}}\right| 0\right\rangle\right)\right|^{2},
$$




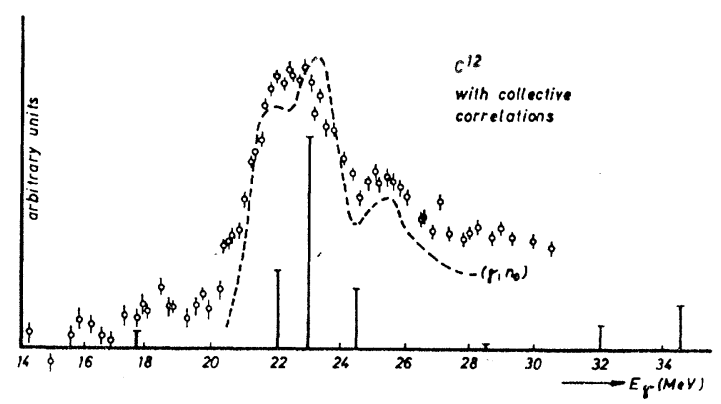

Fig. 8. Photoabsorption cross section of ${ }^{12} \mathrm{C}$. The experimental points give the total photoabsorption cross section; the dashed line gives the $\left(\gamma, n_{0}\right)$ cross section at $90^{\circ}$ in arbitrary units. The dipole strengths are calculated with collective correlations.

where $a_{i}$ are the amplitudes of the pure $1 p$ - $1 h$ states (i.e., no phonon excited) in the eigenstate $|n\rangle$.

\section{RESULTS AND DISCUSSION}

In this section we present the results obtained for ${ }^{12} \mathrm{C},{ }^{28} \mathrm{Si}$, and ${ }^{60} \mathrm{Ni}$ and compare them with experimental data. We approximate the low-energy spectrum of these nuclei by the phonon spectrum of a harmonic oscillator, i.e., we neglect the strong anharmonic terms which split the two-phonon triplet.

In the case of $\mathrm{Si}^{28}$ it might even be worthwhile to repeat the calculations using a deformed basis. Nevertheless, it seems interesting to test the present theory also for light nuclei, although the model seems to be more justified in the case of medium heavy nuclei. It is necessary to say a few words on the parameters entering the calculations. First of all, there are the parameters $B_{2}$ and $C_{2}$ of the harmonic quadrupole oscillator (2). These are taken from the low-energy spectrum. The first excited $2^{+}$state of an even-even vibrational nucleus is interpreted as the one-phonon state of the harmonic surface vibrations. Consequently, its energy is given by $\hbar \omega_{2}=\hbar\left(C_{2} / B_{2}\right)^{1 / 2}=E\left(2^{+}\right)$, and the transition probability to the ground state $B(E 2) \propto \beta_{0}{ }^{2}=5 \hbar /$ $\left(2 B_{2} \omega_{2}\right)$. From both relations one can easily compute $B_{2}$ and $C_{2}$. The quantity $\beta_{0}{ }^{2}$ is the square of the effective vibrational amplitudes, $\beta_{0}=\left(\left\langle 0\left|\sum_{n} \alpha_{n}^{\dagger} \alpha_{n}\right| 0\right\rangle\right)^{1 / 2}$. It

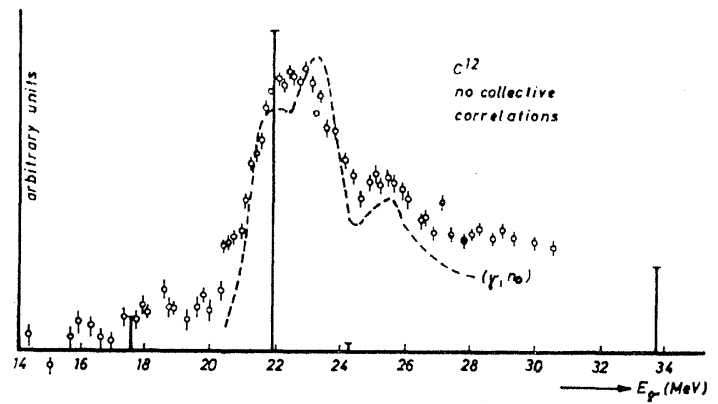

Fig. 9. Photoabsorption cross section of $\mathrm{C}^{12}$. The experimental points give the total photoabsorption cross section; the dashed line gives the $\left(\gamma, n_{0}\right)$ cross section at $90^{\circ}$ in arbitrary units. The dipole strengths are calculated in the usual $1 p-1 h$ model.

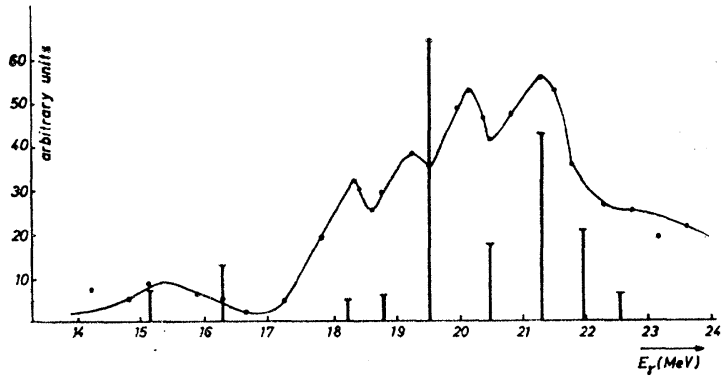

Fig. 10. Results of a particle-hole calculation with collective correlations compared with the experimental absorption cross section.

characterizes the "softness" of the quadrupole vibrations. The symmetry energy parameter of the BetheWeizsäcker mass formula is taken to be $\kappa=20 \mathrm{MeV}$ for all nuclei. The nucleon-nucleon force is of the form

$$
\begin{aligned}
V\left(\mathbf{r}_{1}, \mathbf{r}_{2}\right) & =V 0 f\left(\left|\mathbf{r}_{1}-\mathbf{r}_{2}\right|\right) \\
& \times\left\{a_{0}+a_{\sigma}\left(\boldsymbol{\sigma}_{1} \cdot \sigma_{2}\right)+a_{\tau}\left(\tau_{1} \cdot \tau_{2}\right)+a_{\sigma \tau}\left(\boldsymbol{\sigma}_{1} \cdot \mathbf{\sigma}_{2}\right)\left(\tau_{1} \cdot \tau_{2}\right)\right\} .
\end{aligned}
$$

In actual calculations we use the exchange mixture determined by Gillet ${ }^{5}$ for ${ }^{12} \mathrm{C}$. Therefore, there is only one free parameter, the strength $V_{0}$ of the residual force, which is adjusted so that the energy of the main giant resonance agrees with experiment. We now discuss the results of our calculation for specific nuclei.

\section{A. Giant Resonance Structure of ${ }^{12} \mathrm{C}$}

The low-energy spectrum of ${ }^{12} \mathrm{C}$ shows a $2^{+}$state at $4.43 \mathrm{MeV}$. Recent electron-scattering experiments give $B(E 2)=44 \mathrm{fm}^{4}$ and a transition radius of about $3.3 \mathrm{fm}$; thus $\beta_{0}$ is about $0.43 .{ }^{21}$ For the nucleon-nucleon force, a Gaussian shape with a strength $V_{0}=-35 \mathrm{MeV}$ has been used. The two-phonon states of the surface vibrations can probably be identified with the $7.65-\mathrm{MeV}$ $0^{+}$state and a $2^{+}$state in the $10-\mathrm{MeV}$ region. Thus it seems worthwhile to interpret $\mathrm{C}^{12}$ as a vibrator. The splitting of the 7.65- and $10-\mathrm{MeV}$ states indicates that the contribution of anharmonic terms is appreciable. They are, however, neglected in the present treatment.

The results are shown in Fig. 8 together with the

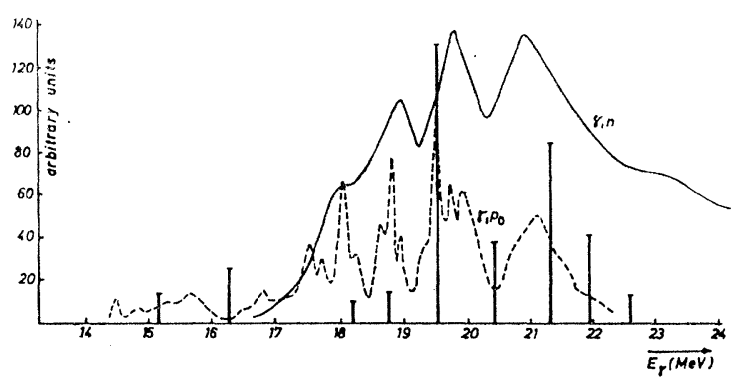

Fig. 11. Results of a particle-hole calculation with collective correlations compared with the $(\gamma, n)$ and $\left(\gamma, p_{0}\right)$ cross sections.

${ }^{21} \mathrm{~F}$. Gudden (private communication). 


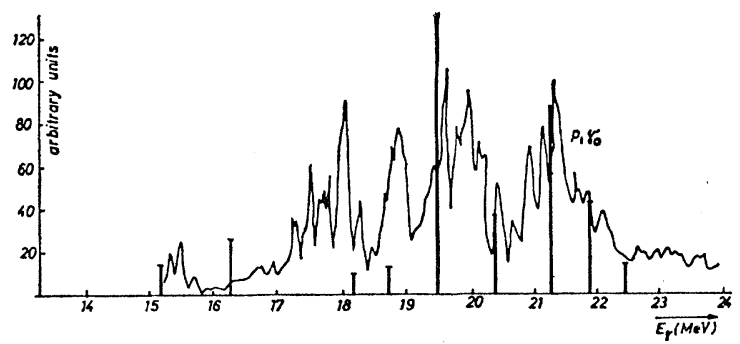

Fig. 12. Results of a particle-hole calculation with collective correlations compared with $\left(p, \gamma_{0}\right)$ cross section.

experimental data of the total photoabsorption cross section and the $\left(\gamma, n_{0}\right)$ cross section. ${ }^{1,22}$ The double peak at 22 and $23 \mathrm{MeV}$ is explained by the theory, as is the strong state at $25.5 \mathrm{MeV}$. However, the predicted energy of the latter state is about $1 \mathrm{MeV}$ too low, although its strength is in agreement with the experiments. Possibly the anharmonic terms of the collective potential, which are neglected in the present calculations, are responsible for this discrepancy. Attention should also be given to the minor states at 18 and 28.5 $\mathrm{MeV}$, which also seem to be indicated in experiments. For comparison with older calculations, we show the results of a pure $1 p-1 h$ calculation in Fig. 9.

\section{B. Giant Resonance Structure of ${ }^{28} \mathrm{Si}$}

In ${ }^{28} \mathrm{Si}$, the first excited $2^{+}$state lies at $1.78 \mathrm{MeV}$. The effective vibrational amplitude $\beta_{0}=0.40$ is known from Coulomb excitation. Again, we approximate the lowenergy spectrum by the harmonic surface vibrator. This seems to be a very crude approximation, since ${ }^{28} \mathrm{Si}$ more closely resembles a deformed nucleus. However, at least for the $1 p-1 h$ part of the calculations, it has turned out that calculations with a deformed basis ${ }^{23}$ do not give an appreciable improvement on calculations with a spherical basis. ${ }^{24}$ The theoretical results are obtained with a strength of the residual force $V_{0}=-60$ $\mathrm{MeV}$. The particle-hole configurations and energies are the same as those of Bolen and Eisenberg..$^{24}$ The results of the calculation, together with various experimental data, are shown in Figs. 10-12. One notices that all major resonances in the experiments may be explained

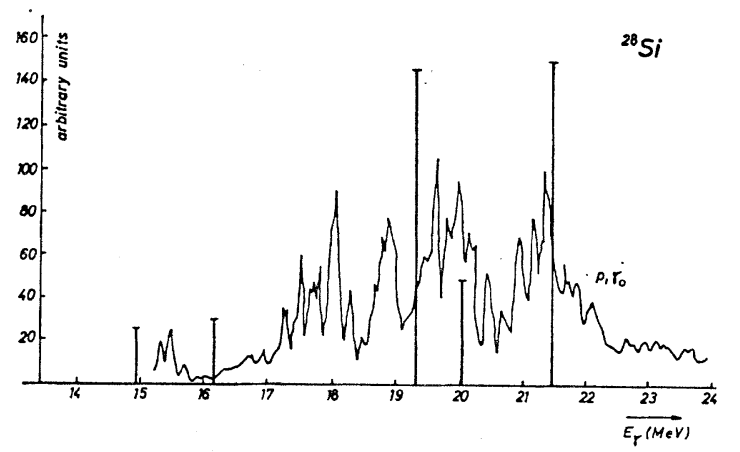

FIG. 13. Results of a $1 p-1 h$ calculation compared with the $\left(p, \gamma_{0}\right)$ cross section.

nearly quantitatively as collective intermediate structure. Especially in the $\left(\gamma, p_{0}\right)$ and $\left(p, \gamma_{0}\right)$ data, however, there is also an indication of noncollective structure. For example, the major peaks in the $\left(p, \gamma_{0}\right)$ cross section at $18.2,18.8,19.6,20.4,21.3,21.9$, and $22.7 \mathrm{MeV}$ are typical for collective intermediate structure. All the fine structure around these resonances is interpreted as noncollective substructure (see Fig. 5). Some disagreement in the energy position of the $15.2-$ and $16.2-\mathrm{MeV}$ states is probably due to inaccurate particle-hole energies for the states involved.

In Fig. 13, the comparison of the experimental data with a pure particle-hole calculation is shown. ${ }^{24}$ The peaks at $19.4,20.1$, and $21.6 \mathrm{MeV}$ represent what is called the $1 p-1 h$ or doorway structure in Fig. 5. The comparison of these results with the calculations shown in Figs. 10-12 clearly indicates the hierarchy of nuclear structures $(1 p-1 h$ doorway, collective intermediate, and noncollective structure) as well as the improvement in the agreement between theory and experiment obtained by the inclusion of collective correlations.

Finally, we show in Fig. 14 the results for different numbers of surface phonons taken into account. The full lines indicate the dipole strengths if four phonons are considered and the dashed lines those for six phonons. It is very satisfactory that the states below $25 \mathrm{MeV}$ are not appreciably affected by this change, in view of the fact that recent particle-hole calculations

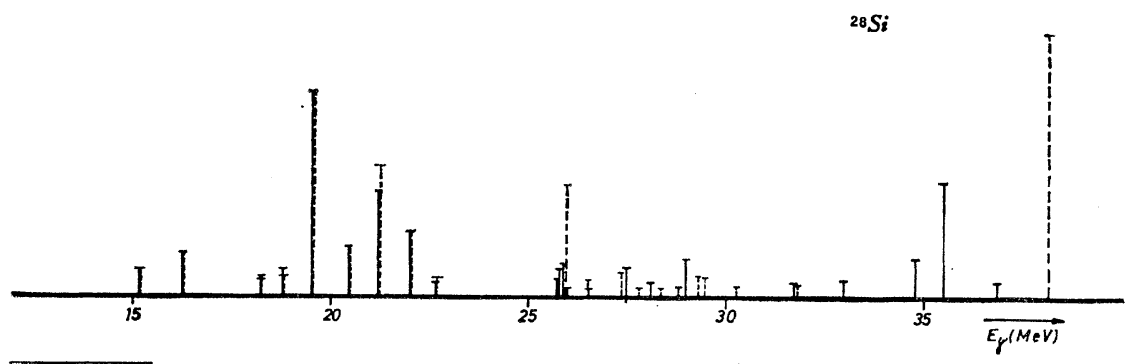

FIG. 14. The influence of higher quadrupole phonons is shown. The levels indicated by full lines are obtained from a calculation where four phonons are included; those indicated by dashed lines were obtained with six phonons.

${ }^{22}$ V. V. Verbinski and J. C. Courtney, Nucl. Phys. 73, 398 (1965)

${ }^{23}$ W. H. Bassichis and F. Scheck, Phys. Rev. 145, 771 (1966).

${ }^{24}$ L. N. Bolen and J. M. Eisenberg, Phys. Letters 9, 52 (1964). 


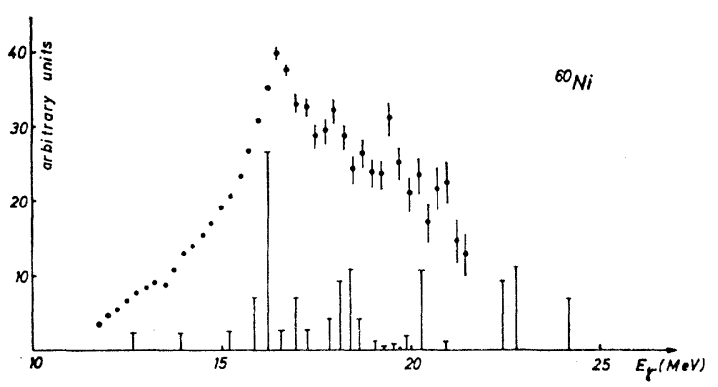

Fig. 15. Relative dipole strengths of eigenstates of ${ }^{60} \mathrm{Ni}$ with collective correlations. The experimental points are from the $(\gamma, n)$ measurements of Ref. 26.

have shown that the "more-phonon states" are usually only poor approximations. ${ }^{25}$

\section{Giant Resonance Structure of ${ }^{60} \mathrm{Ni}$}

The parameters for the collective quadrupole oscillator of ${ }^{60} \mathrm{Ni}$ are $\beta_{0}=0.21$ and $\hbar \omega_{2}=1.33 \mathrm{MeV}$. They are, again, taken from the low-energy spectrum.

The relative strengths of the dipole states obtained from the diagonalization of the energy matrix are presented in Figs. 15-17. Also, for comparison, the experimental $(\gamma, n)$ cross section $^{26}$ for natural nickel $\left(68 \%{ }^{58} \mathrm{Ni}\right.$ and $\left.26 \%{ }^{60} \mathrm{Ni}\right)$ is shown. Of course, the calculated strengths should be compared with the total absorption cross section for the pure $A=60$ isotope.

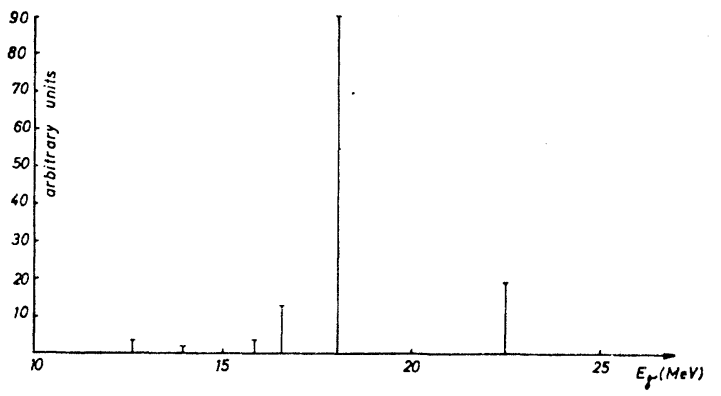

FIG. 16. Dipole strengths of states obtained in the usual particlehole calculation (i.e., without collective correlations).

${ }^{25}$ A. Tokunaga, M. Yamamura, and T. Marumori, On Applicability of the Random-Phase Approximation to the Collective Excitation in Spherical Nuclei (Kyoto University, Japan), Parts I and II.

${ }^{26}$ G. Baciu, G. C. Bonazzola, B. Minetti, C. Molino, L. Pasqualini, and G. Piragino, Nucl. Phys. 67, 178 (1965).

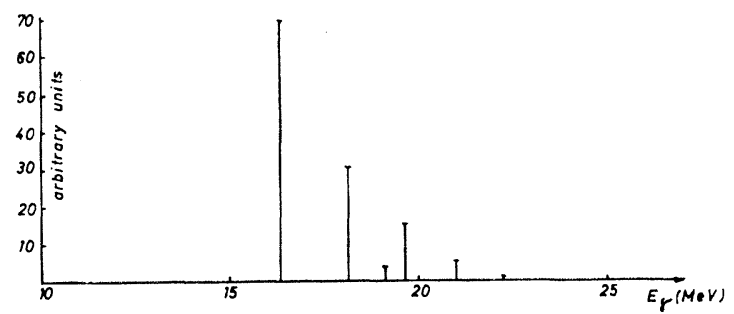

FIG. 17. Relative strengths of dipole states given by the dynamic collective theory.

This is particularly true for nickel inasmuch as the $(\gamma, p)$ cross section is expected to be of the same order of magnitude as the $(\gamma, n)$ cross section.

Until now, however, no such measurements have been reported. Nevertheless, the number of strong dipole states predicted for ${ }^{60} \mathrm{Ni}$, their energies, and their relative strengths are in strikingly good agreement with the available experimental data. Moreover, it is remarkable that so much structure can be nearly quantitatively accounted for with only one adjustable parameter, namely, the strength $V_{0}$ of the nucleon-nucleon force. The results of the pure $1 p-1 h$ calculation (i.e., without collective correlations) are given in Fig. 16. Figure 17 shows the results of the dynamic collective theory (the giant resonances are treated in the collective model). Comparison of Figs. 15-17 indicates that the special features contained in Fig. 16 and Fig. 17 (pure particle-hole structure and pure collective structure, respectively) are also present in Fig. 15. Thus, the extension of the dynamic collective theory introduces additional structure in the giant resonance, in agreement with experiment, and at the same time preserves the general features of the collective theory as well as those of the pure particle-hole description. The merely semiquantitative agreement between theory and experiment indicates, however, that further improvements of the theory are necessary. One of the most important corrections to the present theory of collective correlations are the ground-state correlations. They will be especially important for closed-subshell nuclei.

\section{ACKNOWLEDGMENT}

We are grateful to Dr. M. Danos for many valuable discussions. 